International Journal of Applied Mathematics

Volume 32 No. $6 \quad 2019,959-968$

ISSN: $1311-1728$ (printed version); ISSN: 1314-8060 (on-line version)

doi: http://dx.doi.org/10.12732/ijam.v32i6.5

\title{
EXTREMAL HYPER ZAGREB INDEX FOR TRICYCLIC GRAPHS
}

\author{
Zheng-Qing $\mathrm{Chu}^{1}$, Muhammad Kamran Jamil ${ }^{2} \S$ \\ Aisha Javed ${ }^{3}$ \\ ${ }^{1}$ Department of General Education \\ Anhui Xinhua University, Hefei 230088, CHINA \\ ${ }^{2}$ Department of Mathematics
}

Riphah Institute of Computing and Applied Sciences Riphah International University, Lahore, PAKISTAN

${ }^{3}$ Abdus Salam School of Mathematical Sciences

Government College University, Lahore, PAKISTAN

\begin{abstract}
For a graph $G=(V(G), E(G))$, the first hyper Zagreb index is defined as $\sum_{u v \in E(G)}(d(u)+d(v))^{2}$, where $d(v)$ is the degree of the vertex $v$. The hyper Zagreb index is a kind of extensions of Zagreb index. In this paper, the monotonicity of the hyper Zagreb index under some graph transformations was studied. Using these mathematical properties, the extremal graph among tricyclic graphs are determined for hyper Zagreb index. Moreover, the sharp upper and lower bounds on the hyper Zagreb index of tricyclic graphs are provided.
\end{abstract}

AMS Subject Classification: 05C07, 05C35, 05C40

Key Words: graph transformation; tricyclic graphs; hyper Zagreb index

\section{Introduction}

Let $G$ be a simple, finite and connected graph, with vertex set $V$ and edge set $E$. In a graph $G$ the number of element in $V$ and $E$ is called the order and size, respectively, of $G$. For a vertex $v \in V(G)$, the degree of $v$ is the number of vertices attached to the vertex $v$ and denoted as $d(v)$.

Received: June 17, 2019

(C) 2019 Academic Publications

${ }^{\S}$ Correspondence author 
A topological index of a graph $G$ is a numerical quantity that associated to the graph $G$ with the property that all the graphs isomorphic to $G$ have same quantity. The first and second Zagreb index of a graph $G$ is defined as:

$$
M_{1}(G)=\sum_{v \in V} d(v)^{2} \quad, \quad M_{2}(G)=\sum_{u v \in E} d(u) d(v) .
$$

In 1972, these topological indices appeared for the first time to find the total $\pi$-energy of molecular graphs [9]. Later, the Zagreb indices developed significant applications in QSPR/QSAR studies and a lot of research paper have been published on these, see $[1,2,3,4,5,6]$.

In 2013 Shirdel and co-authors [10] proposed a new version of Zagreb indices called hyper Zagreb index $H M_{1}$. The hyper-Zagreb index is for a graph $G$ defined as:

$$
H M_{1}(G)=\sum_{e \in E} d(e)^{2},
$$

where $d(e)=d(u)+d(v)$ for the edge $e=u v$. Recently, in 2016 Jamil et al. introduced another version of hyper Zagreb index named as second-hyper Zagreb index, defined as

$$
H M_{2}(G)=\sum_{e \sim f} d(e) d(f)
$$

where $e \sim f$ represents that the edges $e$ and $f$ have a common vertex.

Recent results on the hyper Zagreb indices can be seen in $[7,8,11,12,13$, $14,15]$.

We now define some notations which will use in later. Let $\Upsilon_{n}$ denotes the set of all connected tricyclic graphs with order $n$. For a tricyclic graph, the graph which is attained by removing its all pendent vertices is named as a brace of the graph. Denote by $\Upsilon_{n}^{0}$ the set of all braces of tricyclic graphs as depicted in Fig. 6. Let $\Upsilon_{n}^{1}$ and $\Upsilon_{n}^{2}$ denote the sets of tricyclic graphs shown in Figs. 5 and 7 , respectively.

In [8] authors studied the monotonicity of the hyper-Zagreb index with the help of some transformations, they also determined the upper and lower bounds of hyper-Zagreb index on acyclic, unicyclic and bicyclic graphs. In this research paper, we extend the results and find the lower and upper bounds on tricyclic graphs. 


\section{Methods and definitions}

In this section, we introduced some graph transformations, with the help of these transformations we will investigate the extremal graphs with the first hyper-Zagreb index in the set of all connected tricyclic graphs. First we introduced the transformations which strictly increases the first hyper-Zagreb index of a graph.

Transformation 1. Let $u v$ be an edge of connected graph $G$ with $d_{G}(v) \geq$ 2. Assume that $\left\{v, w_{1}, w_{2}, \ldots, w_{t}\right\}$ are all the adjacent to the vertex $u$ while $w_{1}, w_{2}, \ldots, w_{t}$ are pendant vertices. If

$$
K=G-\left\{u w_{1}, u w_{2}, \cdots, u w_{t}\right\}+\left\{v w_{1}, v w_{2}, \cdots, v w_{t}\right\}
$$

we say that $K$ is attained from $G$ by Transformation 1. As shown in Fig. 1.

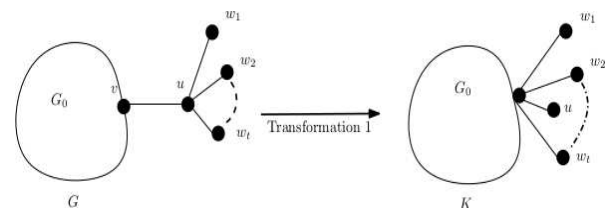

Figure 1: Transformation 1.

Transformation 1 strictly increases the $H M_{1}$ of a graph.

Lemma 1. ([8]) If $K$ is attained from $G$ by Transformation 1 as depicted in Fig. 1, then

$$
H M_{1}(G)<H M_{1}(K) \text {. }
$$

Proof. Clearly, $d_{G}(v)<d_{K}(v)$ and $(d(u)+d(v))$ is not changed during Transformation 1. Hence,

$$
\begin{aligned}
H M_{1}(K)-H M_{1}(G)> & (t+1)\left(d_{G}(v)+t+1\right)^{2}-\left(d_{G}(v)+d_{G}(u)\right)^{2} \\
& -t\left(d_{G}(u)+1\right)^{2} \\
= & (t+1)\left(d_{G}(v)+t+1\right)^{2}-\left(d_{G}(v)+t+1\right)^{2} \\
& -t(t+2)^{2} \\
= & t\left(d_{G}(v)+t+1\right)^{2}-t(t+2)^{2}>0 .
\end{aligned}
$$


Transformation 2. Let $G$ be nontrivial connected graph and $u, v \in$ $V(G)$. Let $P_{a}=(u=) v_{1} v_{2} \cdots v_{a}(=v)$ is a nontrivial $a$-length path of $G$ connecting vertices $u$ and $v$. If $K=G-\left\{v_{1} v_{2}, v_{2} v_{3}, \cdots, v_{a-1} v_{a}\right\}+\{(u+v=$ ) $\left.w v_{1}, w v_{2}, \cdots, w v_{a}\right\}$, we say that $K$ is attained from $G$ by Transformation 2 . As, shown in Fig. 2.

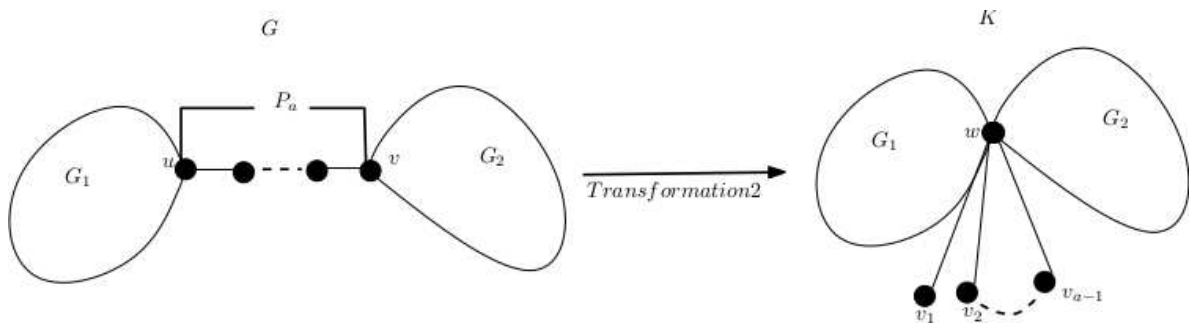

Figure 2: Transformation 2.

Lemma 2. ([8]) If $K$ is obtained from $G$ by Transformation 2 as depicted in Fig. 2, then

$$
H M_{1}(K)>H M_{1}(G) .
$$

Proof. From Fig. 2, let $d_{G_{1}}(u)=x$ and $d_{G_{2}}(v)=y$ while $w=u+v$ (merge $u$ and $v$ to obtain $w$ ) with $d_{K}(w)=x+y+a-1$, where $a \geq 2$. If $a=2$,

$$
H M_{1}(K)-H M_{1}(G)>(x+y+2-1+1)^{2}-(x+y+2)^{2}=0 .
$$

If $a \geq 3$,

$$
\begin{aligned}
H M_{1}(K)-H M_{1}(G)> & (a-1)(x+y+a-1+1)^{2}-(x+3)^{2} \\
& -(y+3)^{2}+16(a-3) \\
= & (a-1)(x+y+a)^{2}-(x+3)^{2}-(y+3)^{2} \\
& -16(a-3) \\
> & (x+y+a)^{2}-(x+3)^{2}+(x+y+a)^{2} \\
& -(y+3)>0 .
\end{aligned}
$$

Transformation 3. Let $H$ be a nontrivial acyclic subgraph of $G$ with $|H|=t$ which is attached at $u_{1}$ in graph $G, u$ and $v$ be two neighbors of $u_{1}$ 

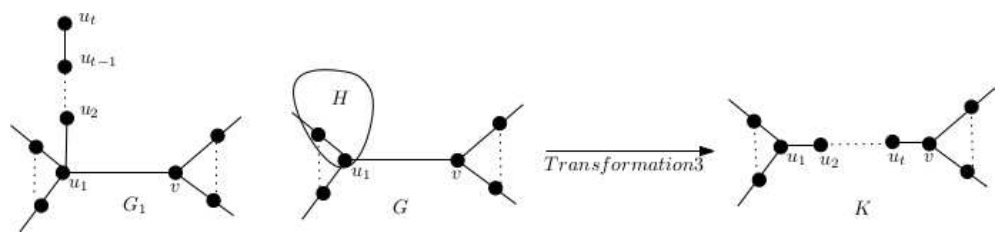

Figure 3: Transformation 3.

different from in $H$. If $K=G-\left(H-u_{1}\right)+u_{1} u_{2}+u_{2} u_{3}+\cdots+u_{t} v$, we say that $K$ is obtained from $G$ by Transformation 3. As shown in Fig. 3.

Lemma 3. Let $G$ and $K$ be graphs, as depicted in Fig. 3, then

$$
H M_{1}(G)>H M_{1}(K)
$$

Proof. From Transformation 1 we know $H M_{1}(G) \geq H M_{1}\left(G_{1}\right)$. So, we only prove the following inequality:

$$
H M_{1}\left(G_{1}\right)>H M_{1}(K)
$$

By definition of $H M_{1}$,

$$
\begin{aligned}
H M_{1}\left(G_{1}\right)-H M_{1}(K)= & \left(d_{G_{1}}\left(u_{t-1}\right)+d_{G_{1}}\left(u_{t}\right)\right)^{2} \\
& +\left(d_{G_{1}}\left(u_{1}\right)+d_{G_{1}}\left(u_{2}\right)\right)^{2} \\
& +\left(d_{G_{1}}\left(u_{1}\right)+d_{G_{1}}(v)\right)^{2} \\
& -\left(d_{K}\left(u_{t-1}\right)+d_{K}\left(u_{t}\right)\right)^{2} \\
& -\left(d_{K}\left(u_{t}\right)+d_{K}(v)\right)^{2} \\
& -\left(d_{K}\left(u_{1}\right)+d_{K}\left(u_{2}\right)\right)^{2} \\
= & \left(d_{G_{1}}\left(u_{1}\right)+2\right)^{2}+\left(d_{G_{1}}\left(u_{1}\right)+d_{G_{1}}(v)\right)^{2} \\
& -\left(d_{G_{1}}\left(u_{1}\right)+1\right)^{2}-\left(d_{G_{1}}(v)+2\right)^{2}-7>0 .
\end{aligned}
$$

This completes the proof.

Let $G$ be a nontrivial connected graph. Two vertices $u$ and $v$ are said to be equivalent, if $G-u \cong G-v$. Clearly, $|N(u)|=|N(v)|$ and their neighbors have the same degree sequence.

Transformation 4. Let $G_{0}$ be a nontrivial connected graph and $u$ and $v$ are two vertices in $G_{0}$ with $d_{G_{0}}(u)=x, d_{G_{0}}(v)=y$ and $N_{G_{0}}(u) \subseteq N_{G_{0}}(u)$. Let 
$G$ be the graph obtained by attaching $S_{k+1}$ and $S_{l+1}$ at the vertices $u$ and $v$ of $G_{0}$, respectively. If $K$ is the graph attained by removing the $l$ pendent vertices at $v$ in $G$ and connecting them to $u$ of $G$, as shown in Fig. 4. We say that $K$ is obtained from $G$ by Transformation 4 .
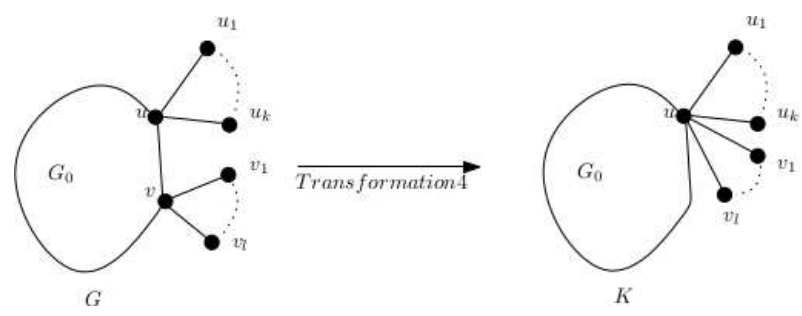

Figure 4: Transformation 4.

Lemma 4. If $\mathrm{K}$ is attained from $G$ by Transformation 4 as depicted in Fig. 4, then

$$
H M_{1}(G)<H M_{1}(K)
$$

Proof. Since, $d_{G_{0}}(u)=x, d_{G_{0}}(v)=y$ and $N_{G_{0}}(v) \subseteq N_{G_{0}}(u)$. So by the definition of $H M_{1}$,

$$
\begin{aligned}
H M_{1}(K)-H M_{1}(G) & =\sum_{i=1}^{k}\left[d_{K}^{2}\left(u u_{i}\right)-d_{G}^{2}\left(u u_{i}\right)\right] \\
& +\sum_{i=1}^{l}\left[d_{K}^{2}\left(u v_{i}\right)-d_{G}^{2}\left(v v_{i}\right)\right] \\
& +\sum_{w \in N_{G_{0}}(v)}\left[d_{K}^{2}(u w)+d_{K}^{2}(v w)\right] \\
& -\sum_{w \in N_{G_{0}}(v)}\left[d_{G}^{2}(u w)+d_{G}^{2}(v w)\right] \\
= & (k+l)(k+l+x)^{2}-k(k+x)^{2}-l(l+y)^{2} \\
+ & \sum_{w \in N_{G_{0}}(v)}\left[\left(k+l+x-d_{G_{0}}(w)\right)^{2}+\left(y+d_{G_{0}}(w)\right)^{2}\right]
\end{aligned}
$$

$$
\sum_{w \in N_{G_{0}}(v)}\left[\left(k+x+d_{G_{0}}(w)\right)^{2}\right.
$$




$$
\left.+\left(l+y+d_{G_{0}}(w)\right)^{2}\right]>2 l(k+x-y)>0 .
$$

This completes the proof.

\section{Results and discussion}

In this section, we found the extremal graph for the first hyper Zagreb index $H M_{1}$ among all tricyclic graphs. To obtain the required result we will use the above discussed transformations.

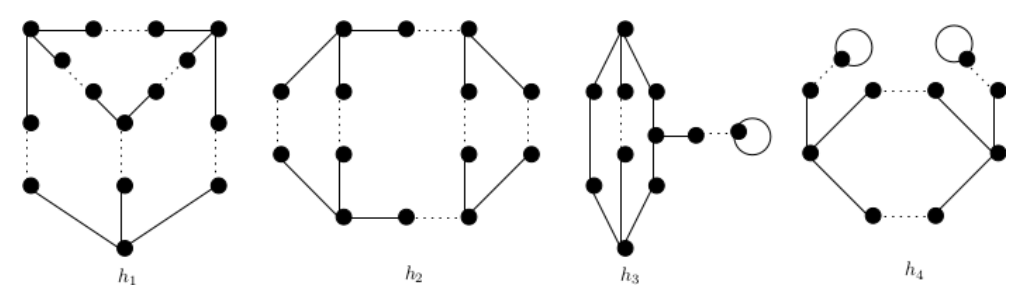

Figure 5: The $h_{i}$ graphs in $\Upsilon_{n}^{1}$.

Theorem 5. Let $\mathrm{G}$ be any tricyclic graph with $\mathrm{n}$ vertices, then

$$
16 n+140 \leq H M_{1}(G),
$$

where the equality holds if and only if $G \in \Upsilon_{n}^{1}$.

Proof. Let $G$ be a connected tricyclic graph. By Lemma 2, $G$ can be converted to the one of the fifteen braces shown in Fig. 6. Meanwhile, for every graph $G$ there exists a graph $g_{i} \in \Upsilon_{n}^{0}$, where $i \leq 15$, such that $H M_{1}\left(g_{i}\right) \leq$ $H M_{1}(G)$ by 3 . Clearly,

$$
H M_{1}\left(h_{i}\right)=16 n+140, \text { for } i=1,2,3,4,5 .
$$

So, the proof is completed.

Theorem 6. Let $G$ be a tricyclic graph of order $n$. Then

$$
H M_{1}(G) \leq n^{3}-n^{2}+12 n+120,
$$

where the equality holds if and only if $G \cong S_{n}^{n+2}$ or $S_{n}^{K_{4}}$. 


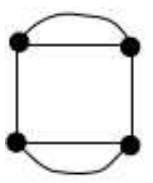

$g_{1}$

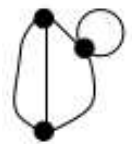

96

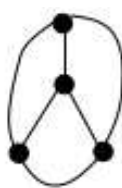

$g_{2}$

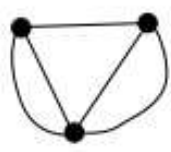

$g_{3}$

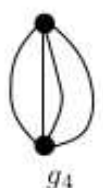

$g_{4}$
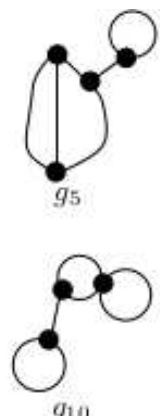

$g_{9}$

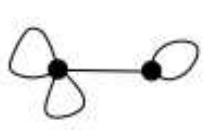

$g_{14}$

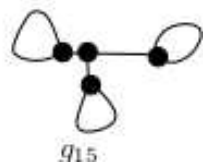

$g_{15}$

Figure 6: The $g_{i}$ graphs in $\Upsilon_{n}^{0}$
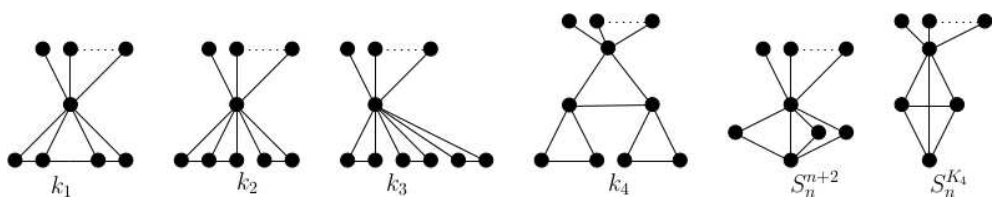

Figure 7: Some graphs.

Proof. For tricyclic graph $G$ with given order $n$ can be transformed to the one of the five graphs shown in Fig. 5 by repeated Transformation 2I and Transformation 4. In other words, for any tricyclic graph $G$ with given order $n$ there exists $k_{i} \in \Upsilon_{n}^{2}$, where $i \leq 6$ such that $H M_{1}(G) \leq H M_{1}\left(g_{i}\right)$ by Lemmas 2 and 4. Notice that,

$$
\begin{gathered}
H M_{1}\left(k_{1}\right)=n^{3}-n^{2}+12 n+96, \\
H M_{1}\left(k_{2}\right)=n^{3}-n^{2}+12 n+74, \\
H M_{1}\left(k_{3}\right)=n^{3}-n^{2}+12 n+54, \\
H M_{1}\left(k_{4}\right)=n^{3}-13 n^{2}+68 n+130, \\
H M_{1}\left(S_{n}^{n+2}\right)=n^{3}-n^{2}+12 n+120, \\
H M_{1}\left(S_{n}^{K}\right)=n^{3}-n^{2}+12 n+120 .
\end{gathered}
$$

Hence, the proof is completed. 


\section{Acknowledgements}

The authors would like to express their sincere gratitude to the Natural Science Foundation for the Higher Education Institutions of Anhui Province of China (nos. KJ2019A0875 and KJ2019A0876), the Quality Engineering Projects of Anhui Xinhua University of China (no. 2017jxtdx05).

\section{References}

[1] B. Bollobàs, P. Erdös, Graphs of extremal weights, Ars Combin., 50 (1998), 225-233.

[2] J. Devillers, A.T. Balaban (Eds.), Topologicial Indices and Related Descriptors in QSAR and QSPR, Gordon and Breach, Amsterdam (1999).

[3] I. Gutman, K.C. Das, The first Zagreb index 30 years after, MATCH Commun. Math. Comput. Chem., 50 (2004), 83-92.

[4] K.C. Das, I. Gutman, Some properties of of the second Zagreb index, MATCH Commun. Math. Comput. Chem., 52 (2004), 103-112.

[5] Y. Gao, M. Imran, M.R. Farahani, H.M.A. Siddiqui, Some connectivity indices and Zagreb index of honeycomb graphs, Int. J. Pharm. Sci. Res., 9, No 5 (2018), 2080-2087.

[6] I. Gutman, M.K. Jamil, N. Akhter, Graphs with fixed number of pendent vertices and first Zagreb index, Trans. on Combinatorics, 4 (2015), 43-48.

[7] W. Gao, M.K. Jamil, M.R. Farahani, The hyper Zagreb index and some graph operations, J. Appl. Math. Comput., 54, No 1-2 (2017), 263-275.

[8] W. Gao, M.K. Jamil, A. Javed, M.R. Farahani, S. Wang, J.B. Liu, Sharp bounds of the hyper-Zagreb index on acylic, unicyclic and bicyclic graphs, Discrete Dynamics in Nature and Society, 2017 (2017), Article ID 6079450, 5 pp.; DOI: doi.org/10.1155/2017/6079450.

[9] I. Gutman, N. Tirnajstic̀, Graph theory and molecular orbitals. III. Total pi-electron energy bydrocarbons, Chem. Phys. Lett., 17 (1972), 535-538.

[10] G.H. Shirdel, H. Rezapor, A.M. Sayadi, The hyper-Zagreb index of graph operatiions, Iranian J. of Mathematical Chemistry, 4, No 2 (2013), 213220. 
[11] M.R. Farahani, The hyper-Zagreb index of Benzenoid series, Frontiers of Mathematics and Its Applications, 2, No 1 (2015), 1-5.

[12] W. Gao, M.R. Farahani, The hyper-Zagreb index for an infinite family of nanostar dendrime, J. of Discrete Mathematical Sciences and Cryptography, 20), No 2 (2017), 515-523.

[13] M.R. Farahani, W. Gao, M.R.R. Kanna, P.R. Kumar, J.B. Liu, General Randic, sum-connectivity, hyper-Zagreb and harmonic indices, and harmonic polynomial of molecular graphs, Advances in Physical Chemistry, 2016 (2016), Article ID 2315949, 6 pp., doi: 10.1155/2016/2315949.

[14] M. Rezaei, W. Gao, M.K. Siddiqui, M.R. Farahani, Computing hyper Zagreb index and mpolynomials of titania nanotubes Tio $2[m, n]$, Sigma J. of Engineering and Natural Sci., 35, No 4 (2017), 707-714.

[15] S. Wang, W. Gao, M.K. Jamil, M.R. Farahani, J. Liu, Bounds of Zagreb indices and hyper Zagreb indices, Mathematical Reports, 21, No 71 (2019), 93-102. 